\title{
Conocimientos, actitudes y prácticas frente a los residuos sólidos de la población universitaria del campus Pance de la Fundación Universitaria Católica Lumen Gentium. Cali, Colombia
}

Knowledge, attitude and practice of the university population concerning solid waste disposal in the Pance campus of the Fundación Universitaria Católica Lumen Gentium. Cali, Colombia

Emilio Latorre*

\section{Resumen}

En esta investigación se estudia el comportamiento de la población universitaria de la Fundación Universitaria Católica Lumen Gentium, en su campus Pance, frente a los residuos sólidos y su manejo. Esto con el propósito de caracterizar dichos comportamientos mediante una encuesta que, usando las bases conceptuales de las representaciones sociales, indaga los conocimientos, actitudes y prácticas de estudiantes, docentes y personal administrativo que opera en el campus. A partir de esta caracterización, se hace una propuesta preliminar de acción, teniendo en cuenta diferentes tipologías de posibles destinatarios de actividades que impulsen cambios de comportamiento, para garantizar el manejo seguro y saludable de los residuos sólidos.

Palabras claves: residuos sólidos, manejo ambiental, basuras, comportamiento humano.

\footnotetext{
* Ingeniero Electricista, Universidad de los Andes, Bogotá. Doctorado en Planeación Regional y Urbana, Universidad de Toulouse 1, Francia. Docenteinvestigador de la Facultad de Ciencias Empresariales de la Fundación Universidad Católica Lumen Gentium. elatorre@Unicatólica.edu.co.

**Administradora de Empresas, Fundación Universidad Católica Lumen Gentium, Cali. Docente-Investigadora de la Facultad de Ciencias Empresariales de la Fundación Universidad Católica Lumen Gentium.
} 


\section{Abstract}

This article studies the behavior of University community of Fundación Universitaria Católica Lumen Gentiumen, Pance campus, in regard solid disposal and its management. The purpose of this research is to characterize those behavior using a social survey applied to students, academic staff and administrative personnel in the campus. Using this characterization, and grouping users of the campus that behave differently in regard solid wastes, a preliminary proposal is presented in order to change their behavior so that their handling are done in a safe and healthy way.

Key words: solid waste, environment management, waste, human behaviour.

\section{Introducción}

Ante una situación cada vez más preocupante sobre el destino de nuestro planeta por causa de los impactos ambientales que le estamos causando, surge la necesidad de interesarnos por cambiar los comportamientos de la sociedad que lo impactan negativamente. En el caso de los residuos sólidos y su manejo, la situación es importante porque su correcta gestión no solo depende de la estructura administrativa que se encarga de su recolección, transporte, utilización y disposición final, sino también del comportamiento de los usuarios y su actuación frente a estos residuos.

En el caso de un campus universitario se espera que exista una buena relación entre estos comportamientos y el área responsable del manejo de los residuos. Pero ese comportamiento depende en gran medida de los modelos mentales que se tengan sobre este tema en la comunidad universitaria. Es aquí donde entran en consideración las representaciones sociales, que son las que enmarcan este trabajo y que se han estudiado a través de una metodología de sondeo denominada CAP (Conocimientos, Actitudes y Prácticas).

Este trabajo muestra el sondeo para tres estamentos universitarios claves: los estudiantes, los docentes y el personal administrativo que se desenvuelven en un campus universitario. Se hace una caracterización de estos aspectos a partir de un cuestionario con 33 preguntas aplicado a 565 personas. Estas preguntas se sintetizaron en siete macro variables, que cobijaron los aspectos de conocimientos, actitudes y prácticas. El estudio muestra en general, un conocimiento importante sobre las acciones a emprender en cuanto a los residuos sólidos y una importante disposición a trabajar a futuro para mejorar esta situación, especialmente por parte de los docentes, personal administrativo $\mathrm{y}$, en menor medida, los estudiantes.
Finalmente, el estudio da cuenta de la disposición de la población estudiada para recibir capacitación y apoyar procesos de educación ambiental frente al manejo de los residuos sólidos en el campus universitario.

\section{Marco teórico}

El concepto de Representación Social fue introducido por Serge Moscovici, un psicólogo francés de origen rumano, en el año de 1961 (Moscovici, 1979). Este planteó que las representaciones de un grupo social no podían resumirse a una sumatoria de las representaciones individuales, y por esa razón "la conciencia colectiva trasciende a los individuos como una fuerza coactiva y que puede ser visualizada en los mitos, la religión, las creencias y demás productos culturales colectivos" (Moscovici, 1979; citado por Mora, 2002, p. 13). Asimismo, Moscovici expresa que “(...) la representación colectiva no se reduce a la suma de las representaciones de los individuos que componen una sociedad" (1979, p. 16).

Moscovici define las representaciones sociales como

(...) Una modalidad particular del conocimiento, cuya función es la elaboración de los comportamientos y la comunicación entre los individuos (...). La representación social es un corpus organizado de conocimientos y una de las actividades psíquicas gracias a las cuales los hombres hacen inteligible la realidad física y social, se integran en un grupo o en una relación cotidiana de intercambios, liberan los poderes de su imaginación (1979, p. 17-18).

En palabras de Mora (2002), las representaciones socialies también pueden ser entendidas como aquel:

Conocimiento de sentido común o bien pensamiento natural (por oposición al pensamiento científico) que se construye a partir de experiencias, informaciones, cono- 
cimientos y modelos de pensamiento recibidos y trasmitidos a través de la tradición, la educación y la comunicación social: un conocimiento socialmente elaborado y compartido (2002, p. 19).

La representación social es, entonces, un modelo mental que es el producto colectivo de la sociedad que interpreta la realidad, en función de lo que observa y escucha. Hoy en día, estas representaciones sociales están muy influenciadas por lo que ofrecen los medios de comunicación, a los cuales están expuestas las personas permanentemente: la televisión, la radio, la publicidad.

Esas representaciones, en últimas, son las que originan o pueden originar comportamientos relacionados con ellas en las personas. Se expresan en cada grupo social de manera verbal, en las conversaciones y opiniones sobre los diferentes fenómenos de la realidad y se traducen en comportamientos. Frente a esto, la representación social “(...) Reconstruye y reproduce la realidad otorgándole un sentido y procura una guía operacional para la vida social, para la resolución de los problemas y conflictos" (Peláez, 1987; , citado por Mora, 2002, p. 8).

De este modo, resulta evidente que las representaciones sociales se convierten en un elemento importante para conocer si un determinado grupo social puede orientar, o no, su comportamiento hacia un manejo adecuado de los residuos sólidos. Para identificar y caracterizar una población frente a sus representaciones sociales relacionadas con los residuos sólidos, se optó por utilizar la metodología de Conocimientos, Actitudes y Prácticas (CAP) en los estamentos universitarios.

Con este fin, el estudio CAP se puede tomar como línea base para conocer el estado inicial de lo que la población tiene como representación social y proyectarlo hacia el futuro. Esto también es muy importante para el diseño de acciones que tengan que ver con cambios de comportamiento o fortalecimiento de acciones de los grupos sociales frente a los temas en estudio. Un estudio CAP indica lo que sabe la gente sobre ese tema, lo que siente y, finalmente, cómo se comporta. Estos tres temas son, entonces, conocimientos, actitudes y prácticas.

Teniendo como base la metodología CAP, se explican de manera breve lo que, por definición, se entiende de cada uno de estos elementos:
Conocimiento: la comprensión de una determinada acción a seguir con respecto a los residuos sólidos (FAO, 2016).

Actitud: las creencias emocionales, motivacionales o perceptivas que tienen una influencia positiva o negativa en el comportamiento o práctica de un individuo (Carruth, 1977; FAO, 2016).

Práctica:la acción observable de un individuo que influye en el manejo de los residuos sólidos (FAO, 2016).

Esta investigación es del tipo exploratorio-descriptivo, ya que muestra cómo se puede caracterizar el comportamiento de los estamentos universitarios frente a un fenómeno que antes no se tenía en cuenta con mucha fuerza en las universidades: el manejo de los residuos sólidos. La información se recoge en mayo de 2016 en su mayoría, y en septiembre de 2016 se realizan algunos complementos faltantes.

\section{Objetivos y metodología}

El estudio se centró en los estamentos universitarios de la Fundación Universitaria $\mathrm{Ca}$ tólica Lumen Gentium (Unicatólica), en su campus de la zona de Pance, en la ciudad de Cali, Colombia. Este campus se encuentra en una zona de parcelaciones al sur de la ciudad, con un ambiente verde y paisajístico privilegiado y con una área aproximada de $25000 \mathrm{mt}^{2}$. Los principales sondeos se hicieron para el mes de mayo del 2016. Para este fin, se tomaron los registros de los estudiantes de los 15 programas académicos vigentes con un total de 4.243 estudiantes en aras de calcular la población a encuestar. Se desarrolló un muestreo estratificado proporcional con un nivel de confianza del $95 \%$, el cual arrojó una muestra de 352 estudiantes, los cuales fueron distribuidos en los estratos, de acuerdo a la representatividad de la población por programa.

Para los docentes se tomaron como referencia los programas de pregrado ofertados en el primer semestre de 2016 y registrados en la página web de la institución. Así, se seleccionaron 21 programas que, mediante muestreo estratificado proporcional y con un nivel de confianza del 
95\%, permitieron identificar una muestra de 184 docentes, los cuales fueron distribuidos proporcionalmente en los estratos de acuerdo a su participación en el total de la población. Para el personal administrativo se estableció una muestra representativa de 29 personas seleccionados de forma aleatoria, con un nivel de confianza del $95 \%$.

A todas estas personas se les aplicó la encuesta (ver Anexo A) que contiene 33 preguntas sobre aspectos de opinión, conocimientos, actitudes y prácticas frente a los residuos sólidos en el campus Pance de Unicatólica.

Se realizó la codificación de las variables, siendo unas de carácter cualitativo y otras cuantitativas, para las cuales se empleó una Escala de Likert (especialmente para actitudes y prácticas). Los conocimientos sobre manejo de residuos sólidos se midieron mediante preguntas con respuesta correcta en el marco de respuestas de múltiple escogencia. Luego de esta codificación, se realizó una captura de datos en formato Excel. La metodología se presenta en la Figura 1.

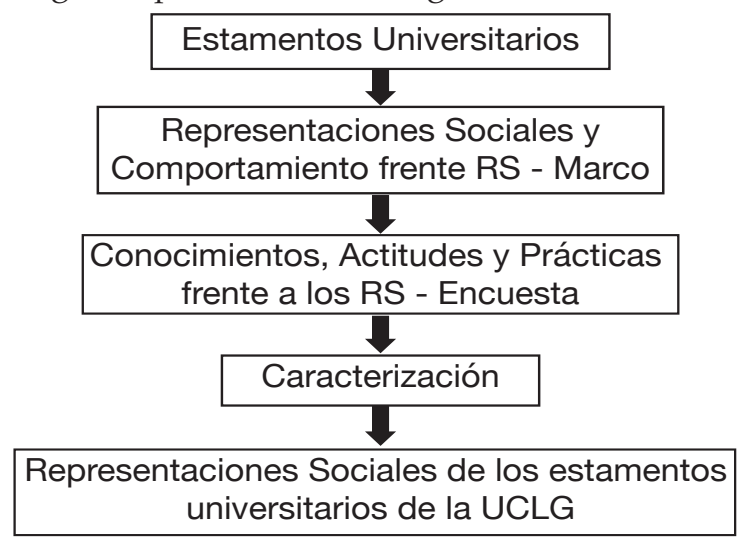

Figura 1 - Metodología general del estudio Fuente: elaboración propia 2017

Con el fin de agrupar los temas de Conocimientos, Actitudes y Prácticas, se crearon siete variables que resumieron las 33 preguntas, como se presenta en la Tabla 1.

Tabla 1.

Variables de agrupación temáticas Conocimiento, Actitudes y Prácticas

\begin{tabular}{|c|c|c|c|c|}
\hline $\begin{array}{c}\text { Variable } \\
\text { Agregada }\end{array}$ & Cap & Descripción & Variables & $\begin{array}{l}\text { Rango y } \\
\text { Relación }\end{array}$ \\
\hline Califica & Conocimiento & $\begin{array}{c}\text { Es la suma de las respuestas correctas a las preguntas } \\
\text { de conocimiento en la encuesta. Si la respuesta es } \\
\text { correcta suma } 1 \text {. Ver Anexo A }\end{array}$ & $\begin{array}{l}\text { Preguntas: P8, P9, } \\
\text { P10, P11, P17, P18, } \\
\text { P19, P29, P30, P33. } \\
\text { Ver las preguntas en } \\
\text { el Anexo A }\end{array}$ & 0 a $10+v a$ \\
\hline $\begin{array}{l}\text { Actitud } \\
\text { hacia el } \\
\text { consumo }\end{array}$ & $\begin{array}{l}\text { Actitud hacia el } \\
\text { consumo }\end{array}$ & $\begin{array}{c}\text { Es la suma de las respuestas donde el menor valor } \\
\text { (2) indica una muy buena actitud hacia el consumo } \\
\text { responsable }\end{array}$ & $\mathrm{P} 28+\mathrm{P} 32$ & 2 a 6 -va \\
\hline $\begin{array}{l}\text { Practicas } \\
\text { responsab. } \\
\text { en el hogar }\end{array}$ & $\begin{array}{l}\text { Práctica en RS en el } \\
\text { hogar }\end{array}$ & $\begin{array}{l}\text { Es la suma de las respuestas donde el valor } 2 \text { indica } \\
\text { una muy buena disposición a tener prácticas } \\
\text { responsables en el hogar con los residuos sólidos }\end{array}$ & $\mathrm{P} 12+\mathrm{P} 25$ & 2 a 6 -va \\
\hline $\begin{array}{l}\text { Prácticas } \\
\text { univ. en } \\
\text { disp. de } \\
\text { residuos } \\
\text { sólidos }\end{array}$ & $\begin{array}{l}\text { Práctica en RS en el } \\
\text { campus }\end{array}$ & $\begin{array}{c}\text { Es la suma de las respuestas donde el valor } 2 \text { es } \\
\text { indica una muy buena disposición a tener prácticas } \\
\text { responsables en la Universidad con los residuos } \\
\text { sólidos }\end{array}$ & $\mathrm{P} 15+\mathrm{P} 20+\mathrm{P} 24+\mathrm{P} 16$ & 4 a 12 -va \\
\hline $\begin{array}{l}\text { Disposic. en } \\
\text { el hogar }\end{array}$ & Actitud & $\begin{array}{l}\text { Buena disposición a acciones responsables frente a los } \\
\text { residuos sólidos en el hogar, donde } 2 \text { es lo mejor }\end{array}$ & $\mathrm{P} 13+\mathrm{P} 27$ & 2 a 8 -va \\
\hline $\begin{array}{l}\text { Dispoc. } \\
\text { a recibir } \\
\text { capac. }\end{array}$ & Actitud & $\begin{array}{c}\text { Buena disposición a recibir capacitación relacionada } \\
\text { con manejo responsable de los residuos sólidos en la } \\
\text { Universidad, donde } 3 \text { es lo mejor }\end{array}$ & $\mathrm{P} 6+\mathrm{P} 21+\mathrm{P} 31$ & 3 a 12 -va \\
\hline $\begin{array}{l}\text { Dispos. } \\
\text { a tomar } \\
\text { acciones }\end{array}$ & Actitud & $\begin{array}{l}\text { Buena disposición a tomar acciones responsables } \\
\text { frente a los residuos sólidos en la Universidad, donde } \\
3 \text { es lo mejor }\end{array}$ & $\mathrm{P} 3+\mathrm{P} 7+\mathrm{P} 22$ & 3 a 12 -va \\
\hline
\end{tabular}

Fuente: elaboración propia 2017 
Cada una de estas variables agregadas se cruzó con las variables de tipo de estamento (estudiantes, docentes, administrativos), genero, edad, facultad, programa académico y semestre (para los estudiantes). Esto permitió realizar un análisis más detallado de la situación CAP. Igualmente, se realizó un estudio particular de las respuestas a ciertas preguntas claves $(8,10,11,17,18,19,20$ $28,29,30$ y 32) que tienen que ver con los conceptos y conocimientos claves sobre manejo de residuos sólidos.

\section{Resultados}

Los resultados se presentan buscando mostrar el comportamiento frente a los residuos sólidos de los distintos estamentos y sus características, teniendo como referencia las siete (7) variables de la Tabla 1. Los factores más importantes que se analizan son:

- Tipo de estamento (estudiantes, docentes y personal administrativo).

- Género (hombres y mujeres).

- Edad

- Estudio especial de la educación frente a los residuos sólidos.

\section{Análisis según tipo de estamento}

En la Figura 2, la cual se orienta a la variable Conocimiento sobre el manejo de los residuos sólidos, se observa una comprensión un poco mayor de los estudiantes frente al manejo de residuos sólidos, ya que $19,1 \%$ de ellos tuvieron, en promedio, 8 o más respuestas correctas a las 10 preguntas sobre este tema que se les formularon. El menor valor lo tuvieron los docentes.

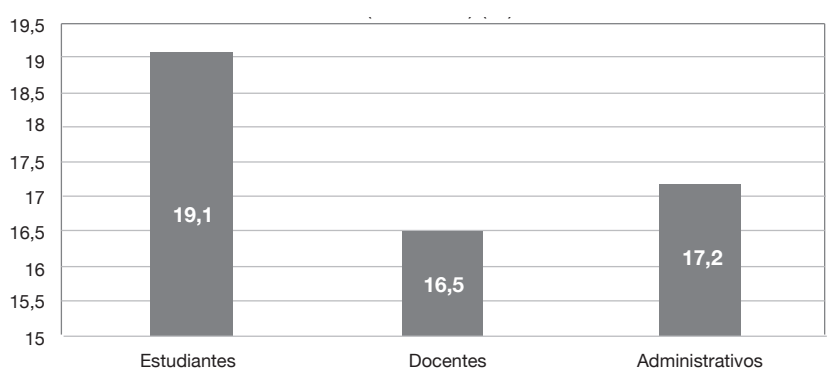

Figura 2. Conocimiento de manejo adecuado de los residuos sólidos de los Estamentos Universitarios del campus Pance Unicatólica 2016 (califica) (\%)

Fuente: UNICATÓLICA (2016). Encuesta CAP: residuos sólidos.
La Figura 3 muestra los resultados de la variable ACTICONSUMO (ver Tabla 1) que señala una mayor actitud de los docentes y administrativos hacia el consumo responsable frente a los residuos sólidos (preguntas 28 y 32, Anexo A), en contraposición con los estudiantes que tuvieron un valor bajo, posiblemente debido a la poca responsabilidad que poseen sobre este tema, por no ser totalmente independiente. Esto se refleja en otras preguntas de la encuesta, en relación con los estudiantes y su manejo de residuos sólidos en el hogar, como por ejemplo en la Figura 4.

Por el contrario, los docentes y los administrativos señalan tener buenas prácticas frente a los residuos sólidos en el hogar (PRACTOGAR, preguntas 5, 31, 35), pero en el campus universitario, las prácticas adecuadas frente a la gestión de residuos sólidos bajan en los tres grupos. Frente a este comportamiento, los docentes señalan tener un mejor comportamiento que los otros dos grupos (preguntas 24 y 7 ).

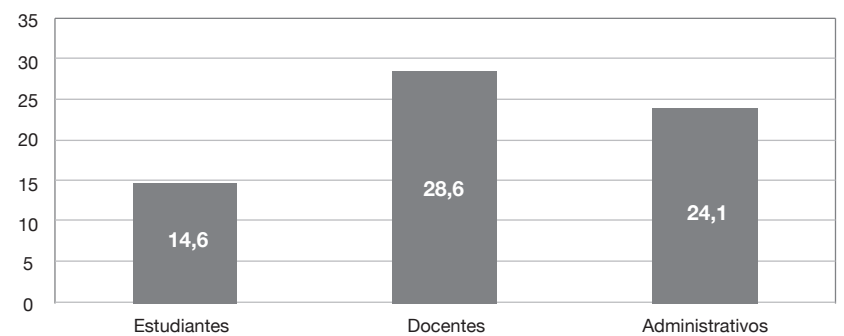

Figura 3. Actitud positiva hacia el consumo sostenible (ACTICONSUMO) de los Estamentos Universitarios del campus Pance - Unicatólica 2016 (\%) Fuente: UNICATÓLICA (2016). Encuesta CAP: residuos sólidos.

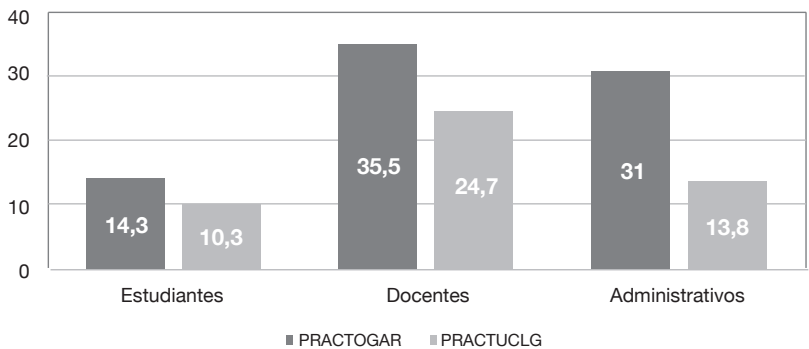

Figura 4. Prácticas de manejo adecuado de los residuos sólidos de los Estamentos Universitarios del campus Pance - Unicatólica en el hogar y en campus $2016(\%)$

Fuente: UNICATÓLICA (2016). Encuesta CAP: residuos sólidos

Una de las preguntas fundamentales para evaluar la disposición hacia la acción en temas de gestión de residuos sólidos en el futuro, se resumen en la Figura 5 que muestran que hay una mayor disposición 
a actuar de los docentes y del personal administrativo, que de los alumnos. Esto indicaría el desarrollo de un trabajo mayor con esta última comunidad.

Los resultados son importantes porque los docentes señalan que desean actuar más en el manejo de los residuos sólidos en el hogar en un $60.1 \%$, que desean recibir capacitación en un 56.3 \%, y que están dispuestos a actuar mejor en la universidad frente a los residuos sólidos en un $58.5 \%$. El personal administrativo desea tener todavía más capacitación (69 \%) que los docentes y está más dispuesto a colaborar en un $65.5 \%$. Hay entonces un importante deseo de colaboración para acciones frente a los residuos sólidos en Unicatólica, pero hay que trabajar más en los estudiantes, y el personal administrativo parece ser un buen aliado.

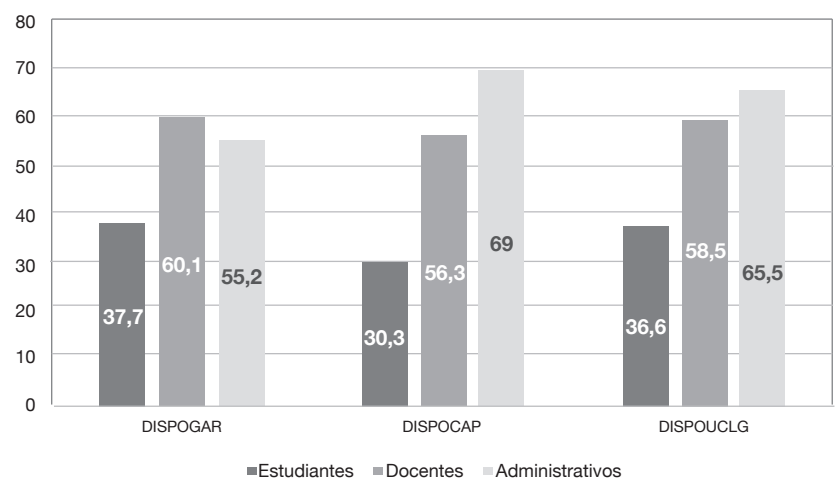

Figura 5. Dispocición a actuar en temas de manejo de residuos sólidos en el hogar (DISPOGAR), en la universidad (DISPOUCLG), y a recibir capacitación (DISPOCAP) de los estamentos universitarios frente a los residuos sólidos campus Pance - Unicatólica 2016

Fuente: UNICATÓLICA (2016). Encuesta CAP: residuos sólidos.

\section{Análisis según género}

En lo relacionado al género, la Figura 6 resume los resultados de Conocimientos, Actitudes y Prácticas frente a los residuos sólidos en los estamentos analizados. En cuanto a la variable conocimientos, las mujeres están un poco por encima de los hombres (CALIFICA), pero en todas las demás variables, están por debajo de ellos. Esto es más marcado en las prácticas en el hogar y en la universidad, y también en la disposición a actuar y a recibir capacitación hacia el futuro, donde las mujeres están ocho puntos porcentuales por debajo de los hombres.

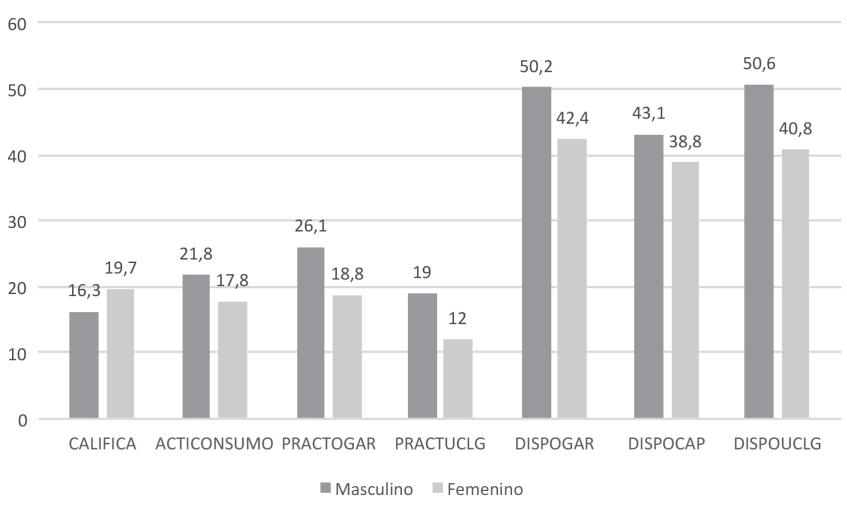

Figura 6. Conocimientos, Actitudes y Prácticas según género frente a los residuos sólidos de los Estamentos Universitarios de Unicatólica 2016 (\%) Fuente: UNICATÓLICA (2016). Encuesta CAP: residuos sólidos.

\section{Los resultados según edad}

La edad no parece ser un factor diferenciador para el conocimiento sobre el actual de las comunidades analizadas frente a la gestión de los residuos sólidos, porque las personas mayores de 41 años tienen más o menos el mismo conocimiento que los jóvenes de 17 a 20 años. Lo que sí es significativo es la actitud hacia el consumo y hacia los residuos sólidos, y la disposición a trabajar en su buen manejo y a recibir capacitación, que es mucho mayor en las personas de más edad.Todas las variables muestran un mayor valor porcentual a medida que aumenta la edad (ver Figuras 7 y 8 ).

Tomando como ejemplo la variable DISPOUCLG, el valor pasa de $29.4 \%$ para los jóvenes de 17 a 20 años, a $65.8 \%$ para los mayores de 41 años. Igualmente, la disposición a recibir capacitación pasa de $22.8 \%$ a 69.9 $\%$ para estos mismo rangos de edades.

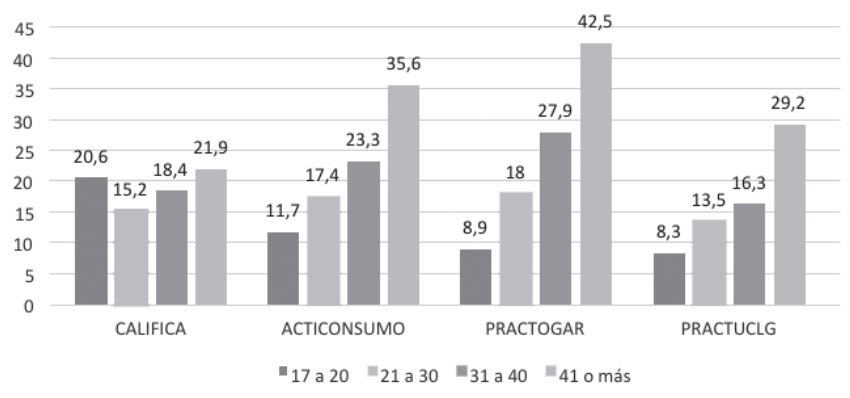

Figura 7. Conocimientos, Actitudes y Prácticas frente a los residuos aólidos de los Estamentos Universitarios de Unicatólica. según edad - 2016 (\%) Fuente:UNICATÓLICA (2016). Encuesta CAP: residuos sólidos. 


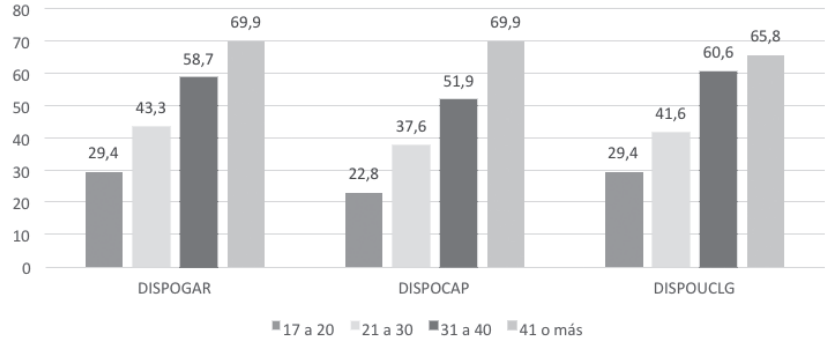

Figura 8. Actitudes frente al manejo de los residuos sólidos de los Estamentos Universitarios de Unicatólica, según edad - 2016 (\%)

Fuente: UNICATÓLICA (2016). Encuesta CAP: residuos sólidos.

\section{Análisis sobre educación en residuos sólidos}

La encuesta orienta varias preguntas al tema de la inclusión de la educación en el manejo de los residuos sólidos, desde la perspectiva de los estamentos universitarios. Las preguntas 4, 5, 14, 23, y especialmente la 26 , tocan este tema (ver Anexo A).

El análisis de las preguntas 4 y 5, relacionado con el hecho de que se realice educación en el tema de los residuos sólidos en la Universidad, muestra que esto es escaso en todos los estamentos y géneros (ver Figura 9). No obstante, se constata el amplio apoyo para que esta iniciativa se desarrolle, especialmente entre docentes y administrativos (71 \% y $82.8 \%$, respectivamente). En cuanto al comportamiento de género, no parece haber mucha diferencia (Figura 10).

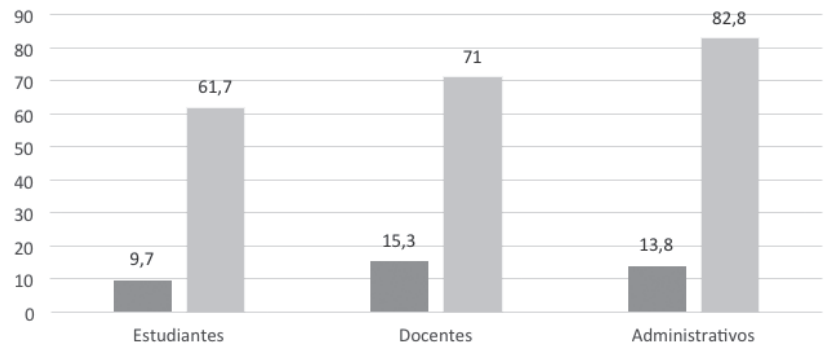

Figura 9. Inclusión del tema de los residuos sólidos en los cursos de UNICATÓLICA según los estamentos, en mayo 2016

Fuente: UNICATÓLICA (2016). Encuesta CAP: residuos sólidos.

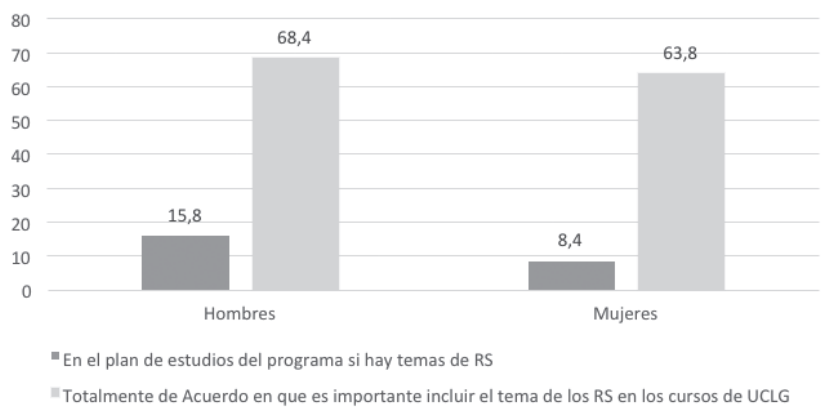

Figura 10. Inclusión del tema de los residuos sólidos en los cursos de UNICATÓLICA según género, en mayo 2016

Fuente: UNICATÓLICA (2016). Encuesta CAP: residuos sólidos.

La Vicerrectoría Académica y la Facultad de Educación muestran que esta capacitación es una prioridad para ellas (100\% y $77.5 \%$ respectivamente) (ver Figura 11). De todas formas, para todas las facultades este porcentaje siempre es mayor al $50 \%$.

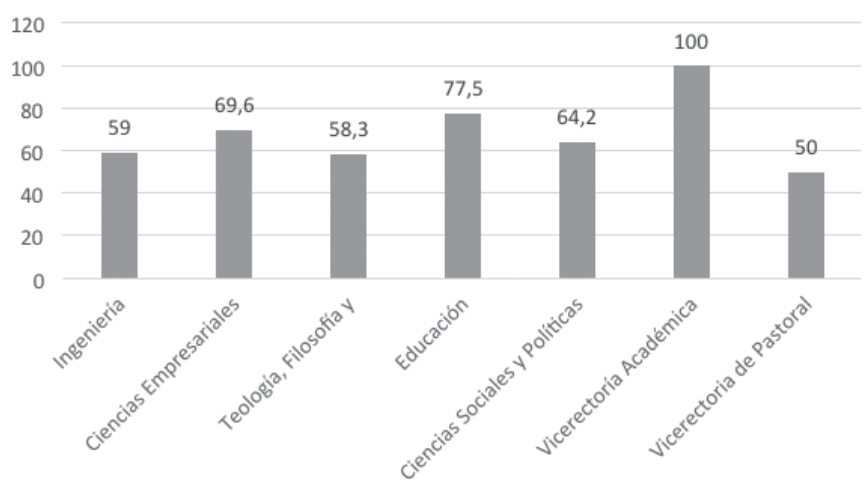

Figura 11. Totalmente de acuerdo en que es importante incluir el tema de los residuos sólidos en los cursos de Unicatólica, según facultad, en mayo 2016

Fuente: UNICATÓLICA (2016). Encuesta CAP: residuos sólidos.

Finalmente, una pregunta que es clave y que se formuló en la encuesta, relacionada con la disposición a hacer parte de campañas para el correcto manejo de los residuos sólidos en la Universidad, tuvo una respuesta aceptable, pero no abrumadoramente mayoritaria, como indica la Figura 12. Con este potencial habría que trabajar, contando con menos apoyo por parte de estudiantes y mujeres, y con un mayor apoyo de la Facultad de Educación y de la Vicerrectoría Académica (ver Figura 13). 


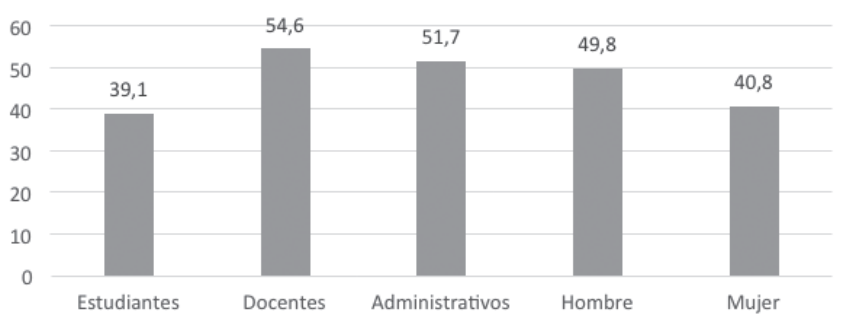

Figura 12. Totalmente de acuerdo en estar dispuesto a ser parte de campañas para sensibilizar en cuanto a la correcta gestión de residuos sólidos

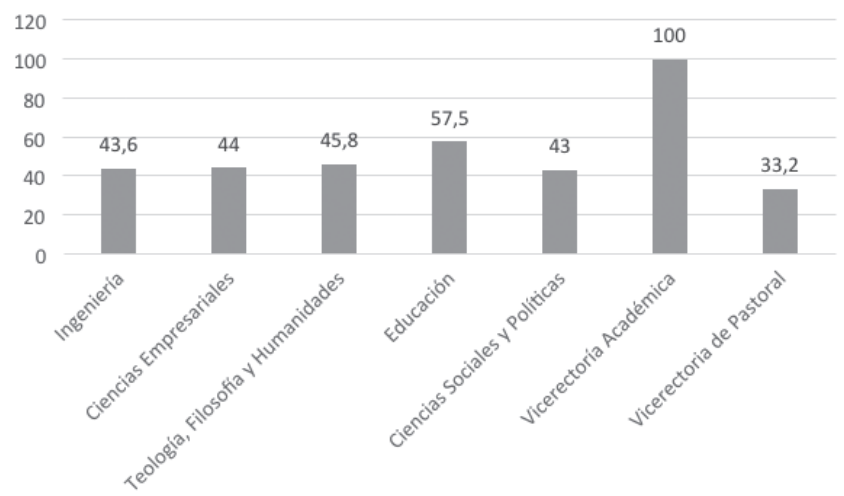

Figura 13. Totalmente de acuerdo en estar dispuesto a ser parte de campañas para sensibilizar en cuanto a la correcta gestión de residuos sólidos Fuente: UNICATÓLICA (2016). Encuesta CAP: residuos aólidos.

\section{Análisis focalizado en las representaciones sociales}

En el interés de estudiar las representaciones sociales de los estamentos universitarios frente a los residuos sólidos, se seleccionaron algunas preguntas claves que ayudan a entender aquellas imágenes que existen en las personas, y que están orientando sus actitudes y actuaciones en el tema de la investigación que nos ocupa. Esas preguntas fueron:

8. ¿Qué entiende por residuos sólidos?

10. La principal característica de los residuos sólidos es que son ...

11. De acuerdo a su conocimiento, separar en la fuente es...

17. Si va a depositar residuos como papel, cartón, periódicos, ¿en la caneca de cuál color lo haría?

18. Si va a depositar residuos como envases plásticos no retornables, bolsas plásticas, desechables plásticos, ¿en la caneca de cuál color lo haría?

19. Si va a depositar residuos como envolturas de alimentos, papel sucio y engrasado, servilletas sucias, papel aluminio, papel carbón, ¿en la caneca de cuál color lo haría?

20. Cuando va a depositar un residuo sólido y encuentra diferentes recipientes, ¿qué hace usted?

28. ¿ Reutiliza usted alguna clase de residuos sólidos?

29. ¿Qué es para usted reciclar residuos sólidos?

30. ¿Qué es para usted reutilizar residuos sólidos?

32. Si usted conoce que el producto que va a comprar es hecho con material reutilizado o reciclado, ¿lo compraría?

El análisis de las respuestas a estas preguntas brinda una buena idea de las representaciones sociales de los encuestados a este respecto.

El análisis de las respuestas a estas preguntas ofrece entonces ideas sobre las representaciones sociales de los encuestados. En primer lugar, para la respuesta relacionada con el concepto de residuos sólidos (pregunta 8), se encuentra entre los encuestados en desuso del término basura, el cual ha sido reemplazado por el concepto de residuo aprovechable (45.8\%). Las mujeres parecen reconocer mejor que los hombres el significado del concepto residuos sólidos (50 $\%$ frente al $41 \%$, respectivamente) (ver Figura 14).

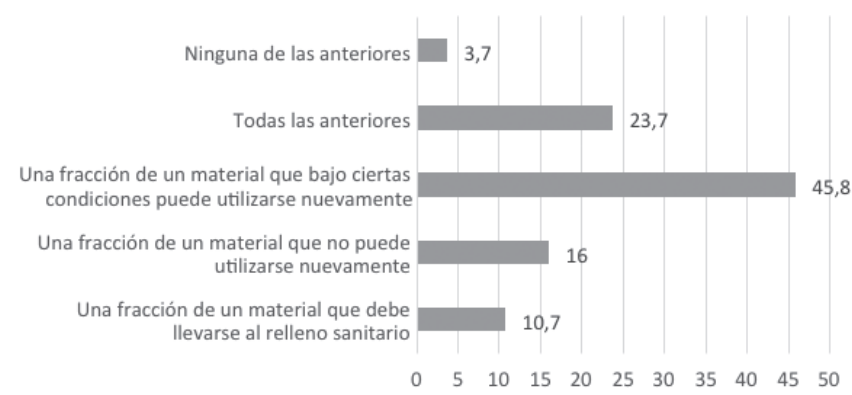

Figura 14. ¿Qué entiende por residuos sólidos? (cifras expresadas en porcentajes)

Fuente: UNICATÓLICA (2016). Encuesta CAP: residuos sólidos.

A la pregunta 10, que es complementaria de la anterior, los encuestados respondieron de manera similar, opinando que la principal característica de los residuos sólidos es que son recuperables/aprovechables (62.5 $\%)$. Sin embargo, el $18.9 \%$ de los encuestados dice no saber la respuesta y el 5.2\% opina que son peligrosos. Esto indica que existen grupos importantes que tienen que ser instruidos aún más en el tema, para que tomen conciencia de que el residuo sólido es un bien susceptible de ser aprovechado (ver Figura 15). 


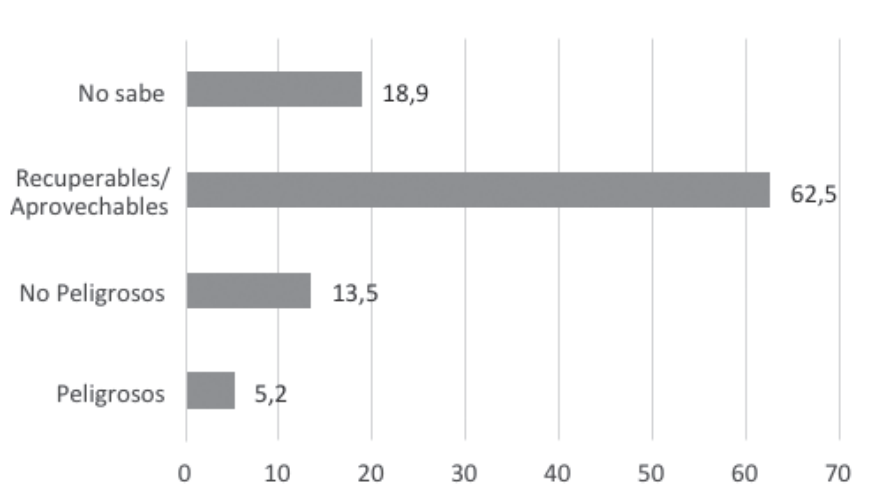

Figura 15. Principal característica de los residuos sólidos (cifras expresadas en porcentajes)

Fuente: UNICATÓLICA (2016). Encuesta CAP: residuos sólidos.

El concepto de separación en la fuente es claro para la mayoría de los encuestados (pregunta 11), pues el $74.4 \%$ lo manejaron con claridad. Nuevamente aquí, un $12.6 \%$ de los encuestados no saben lo que es separar en la fuente, asunto preocupante, pues es un grupo alto, principalmente entre los estudiantes.

El manejo de los colores de las canecas para depositar los residuos sólidos, de acuerdo a sus características, es altamente deficiente. Hay una gran confusión reflejada en las preguntas 17 y 19, al presentar estas bajos niveles de respuestas acertadas por parte de los participantes (19.6 \% y $11.4 \%$, respectivamente). La pregunta 18, relacionada con los residuos plásticos, sí tuvo un mayor nivel de respuestas acertadas, con referencia a las dos preguntas anteriores, ya que el 52.1 $\%$ de los encuestados identificaron la caneca azul.

Este desconocimiento de las canecas y sus colores para depositar los residuos sólidos, contrasta un poco con la respuesta de la pregunta 20, que indaga por el comportamiento de las personas frente a los colores de los recipientes y a su correcta utilización. En efecto, el 50.7 \% señala que se aseguraron del color del recipiente antes de usarlo, según el residuo que iban a depositar en él. De igual manera, hay un $42.2 \%$ de encuestados que respondieron" algunas veces" al hecho de revisar el color de la caneca antes de usarla, y un 7.1 \% asegura nunca tener en cuenta el color de la caneca previo a su uso. Es importante resaltar que dentro de este último grupo se encuentran alto número de estudiantes.

No parece haber una cultura del reutilización de los residuos sólidos, aunque en el contexto universitario esto es difícil. Dado que la pregunta 28 se hizo en tér- minos genéricos, esto cobija lo que se haga tanto en la Universidad, cómo en otros contextos del encuestado (hogar, recreación, ecalle, por ejemplo). Para esta pregunta el nivel es bajo, pues solo el 28.8 \% responde que sí reutilizan los residuos sólidos. Por su parte, el $40.2 \%$ dice nunca reutilizar los materiales.

Los encuestados tienen un correcto concepto del reciclaje, que se observa en las respuestas a la pregunta 29, pero hay que reconocer que las opciones de respuesta son bastante obvias. En cuanto a la reutilización, parece haber menos conocimiento entre las personas, pues solo el $44.3 \%$ acierta la respuesta correcta (volver a usar un determinado producto para darle una segunda vida, con el mismo uso u otro diferente), mientras que el $8.2 \%$ no sabe qué es reutilizar (ver pregunta 30 ).

Los materiales que más reutilizan las personas encuestadas son, en su orden, papel (16\%), botellas plásticas (16 \%) y tapas (12.1\%). No obstante, se presentan valores preocupantes, dado que el $39.9 \%$ de los encuestados no reutiliza los residuos y un 34 $\%$ no sabe o no responde. Aquí se presenta claramente una gran deficiencia (sobre todo en la comunidad estudiantil) frente a una verdadera toma de conciencia sobre al manejo de los residuos sólidos.

La pregunta 32, relacionada con la disposición de los encuestados a trabajar por el medio ambiente en el campo del reciclaje y la reutilización, es desalentadora. Solo el 52.2\% lo haría frecuentemente, mientras que el $41.5 \%$ lo haría algunas veces, y el $6.2 \%$ nunca lo haría.

Al preguntársele la razón que influencia la compra o no de productos a base de material reciclado, para aquellos que respondieron que los comprarían (52.2 $\%)$, se obtuvo lo siguiente:

- El 40 \% lo harían por ayudar al medio ambiente, al planeta y/o a la naturaleza.

- $\quad$ El $17.4 \%$ no sabe / no responde.

- El 15.4 \% lo harían por disminuir la contaminación, reciclar y/o ahorrar.

- El 13\%, porque contribuye a la conservación de los recursos.

- El $9.2 \%$ porque lo necesitamos, me importa y/o me gusta.

- Por su parte, al preguntársele la razón por la cual 
no comprarían un producto a base de material reciclado para aquellos que nunca lo comprarían $(6.2 \%)$, se obtuvo lo siguiente:

- El $40 \%$ porque es antihigiénico, repulsivo y/o dudaría del proceso de manufactura.

- $\quad$ El $14.3 \%$ no sabe / no responde.

- El $11.4 \%$ porque no me importa y/o no me interesa.

- $\quad$ El $2.9 \%$ porque me da pereza.

Estas respuestas son importante,s porque exponen las tendencias del pensamiento colectivo de los estamentos universitarios analizados. Aunque hay un buen acercamiento a los temas ambientales, parecería que hay todavía mucho por hacer para afianzar estas ideas y que el manejo racional y responsable de los residuos sólidos se convierta en un referente colectivo permanente y en una práctica cotidiana.

Todavía se presenta un porcentaje de población que está renuente a estos temas de uso racional de los materiales, y que no orientan su consumo y uso para minimizar los impactos de los residuos sólidos $(46.7 \%)$. Este valor es muy significativo si se tiene en cuenta que esta era una encuesta libre de responder, sin ninguna presión de tiempo, ni de supervisión y, claro está, no se pedía la identificación de la persona.

\section{Conclusiones}

Este trabajo ha permitido caracterizar los estamentos universitarios frente a los residuos sólidos en el campus Pance de la Fundación Católica Lumen Gentium en la ciudad de Cali, Colombia, en el año 2016. Sirvió como marco teórico el concepto de Representaciones Sociales y como instrumento metodológico centrarse en estudiar los Conocimientos, Actitudes y Prácticas de los estamentos frente a los residuos sólidos, denominado estudio CAP. El trabajo se realizó mediante una encuesta aplicada a estudiantes, profesores y personal administrativo de la Universidad en mayo de 2016, principalmente.

Una primera conclusión importante es que el enfoque de las representaciones sociales es muy útil para conocer ese "pensamiento colectivo" que existe frente a muchos asuntos de la vida cotidiana $y$, en este caso en particular, frente a un tema ambiental primordial, como es el de los residuos sólidos.

La concepción de las representaciones sociales mostró su validez en este estudio, máxime asociado al método $\mathrm{CAP}$, para conocer lo que está pasando en un grupo social y poder enfocar acciones para ajustarse a esta realidad y poder proponerle cambios y modificaciones. Es muy útil articular esta metodología con el manejo del mercadeo social como instrumento para'vender'la idea de emprender acciones de beneficio colectivo.

En este sentido este estudio ofrece orientaciones para diseñar acciones y campañas para promover cambios en actitudes y comportamientos de los estamentos de Unicatólica. Tal como dice la Teoría de las Representaciones Sociales, se debe desarrollar un proceso de anclaje, para lograr ese enraizamiento de las ideas fuerza del manejo responsable de los residuos sólidos, mediante las tres funciones básicas de la representación social, que son: 1) la cognitiva (asimilación de una nueva cultura ambiental), 2) la interpretativa de la realidad (conocer el manejo concreto y adecuado de los residuos sólidos), y 3) la orientación de conductas (qué hacer) y de las relaciones sociales alrededor de este manejo de los residuos sólidos (justificar el comportamiento ante los otros).

El trabajo se basó en 33 preguntas formuladas, unas de opinión, otras de conocimientos, otras de actitud y otras de prácticas. Esto orientó las acciones para reagrupar las variables en siete macro variables, que son el resultado de la agregación de variables similares. En cuanto al conocimiento sobre el manejo de los residuos sólidos, los estudiantes tienen un ligero mejor nivel que los otros estamentos.

En cuanto al conocimiento sobre el manejo de residuos sólidos, los estudiantes tienen un nivel ligeramente mejor, que los otros estamentos. En cuanto a una actitud positiva hacia el consumo sostenible, en general los docentes y administrativos tienen un mejor comportamiento. Esto mismo sucede en cuanto a las prácticas adecuadas con los residuos sólidos, tanto en el hogar, como en el campus universitario.

Asimismo, se presenta una buena disponibilidad de docentes y administrativos hacia una capacitación en manejo adecuado de los residuos sólidos. También se evidenica una buena disposición a participar en cam- 
pañas, pero no es generalizada, contando con aproximadamente una participación porcentual del $50 \%$ de la población universitaria.

Esta investigación permite concluir que ya existe entre la población universitaria de la Unicatólica un importante manejo del tema relacionado con residuos sólidos, dado que existen representaciones sociales evidentes de actitudes positivas frente a este tema en la comunidad. Sin embargo, todavía se encuentra un grupo de personas que aún no comparten o no conocen los postulados de la gesitón de residuos sólidos, por lo que también se hace necesario el desarrollo de campañas de refozamiento en este tipo de temáticas.

\section{Recomendaciones}

Esta investigación muestra que hay mucho potencial para trabajar en el tema de residuos sólidos en la Universidad. Podría señalarse, igualmente, que esto toca al tema ambiental en general.

En primer lugar, es fundamental elaborar el PGIRS (Plan de Gestión Integral de Residuos Sólidos) de la Universidad, identificando claramente los siguientes aspectos:

a. Establecer línea de base de los residuos sólidos, que incluya una caracterización actual del volumen de su generación por tipo de residuo, incluyendo producción per cápita.

b. Identificar un sistema de ubicación de los recipientes y contenedores de los residuos sólidos, espacialmente distribuidos en el campus, y de buena accesibilidad para los usuarios.

c. Establecer un sistema de recolección y transporte de residuos sólidos.

d. Organizar el barrido y limpieza de áreas comunes.

e. Corte de césped y poda de árboles, su manejo y disponibilidad.

f. Gestión de residuos de construcción y demolición.

g. Residuos sólidos especiales (peligrosos).

h. Aprovechamiento de los residuos sólidos.

i. Disposición final adecuada.

De acuerdo con este plan, realizar un proceso de educación ambiental que oriente conocimientos y actitudes sobre los residuos sólidos, su manejo y prácticas para reducir, reusar, reciclar y disponer adecuadamente los residuos. Este plan debe seguir unos lineamientos, basados en los principios del Mercadeo Social y podría ser adelantado en colaboración entre: Proyección Social, Servicios Generales y Compras, Comunicaciones y las facultades de Educación y Ciencias Empresariales.

El Plan de Educación en Residuos Sólidos debería ser parte de un programa más amplio de educación ambiental de la Universidad, dentro del marco de un proyecto de Campus Sostenible. Las acciones del Plan de Educación deberían enfocarse en los tres estamentos universitarios, pero teniendo en cuenta los resultados de este proyecto para poder focalizar mejor las acciones.

Antes de adelantar acciones en el tema de campañas y de educación ambiental en residuos sólidos, se deben integrar todos los demás elementos ambientales que entran en la vida universitaria: ahorro en el consumo de agua y energía, alimentación saludable, manejo adecuado del paisaje y de las zonas verdes, transporte sostenible, cero contaminaciones atmosféricas, entre otros.

Un enfoque integral de estos temas supone involucrar el tema ambiental en el currículo de todos los planes de estudio de la Universidad. Tarea difícil, más no imposible. Esto es lo que exige el compromiso ambiental. También aprovechar los espacios de comunicación y dialogo con los alumnos, los docentes y el personal administrativo de la Universidad, para incluir los temas de comportamiento ambiental.

Una vez haya claridad sobre el norte ambiental de la Universidad, se pueden planificar en forma coordinada y periódica las acciones físicas, reglamentarias y educacionales para mejorar la relación del campus con su entorno y para ofrecer un ambiente de estudio y de trabajo sano.

Otra acción importante es la de realizar un estudio especial de la cafetería del campus, para reducir, en forma progresiva, los residuos sólidos derivados de los empaques de los productos que se venden, a la vez que se promueve una alimentación sana y saludable. Esto incluye la disminución de los empaques plásticos que se usan para servir los alimentos, lo que debe ir acompañado de un trabajo conjunto con los concesionarios de las cafeterías y amarrado a campañas ilustrativas a los usuarios de estas mismas, en forma progresiva e informada. 
Finalmente, el estudio ha proporcionado un importante acercamiento a las representaciones sociales asociadas al tema de los residuos sólidos. Se recomienda que para estudios más detallados a nivel de facultades o de grupos sociales, o de género, el material de la encuesta sirva para precisar acciones. Por ello, este queda disponible para trabajos futuros y como línea de base, para hacer comparaciones y mirar tendencias.

\section{Referencias bibliográficas}

Alfonso Pérez, I. (2016). La Teoría de las Representaciones Sociales. Recuperado de http://wwww.psicologia-online.com/ articulos/2007/representaciones sociales.shtml

Bruntland, C. (1987). Nuestro Futuro común. Oxford University Press. Londres.

Carruth, B. (1977). Scaling criteria in developing and evaluating an attiude instrument. Journal of the American Dietetic Association. 70, 1, 42-47.

FAO. (2016). Guidelines for assessing nutrition-related Knowledge, Attitudes and Practices. Recuperado de http://wwww.fao. org/docrep/019/i3545e/i3545e00.htm

Farr, R. M. (1986). Las Representaciones Sociales en: Serge Moscovici (compilador) Psicología Social II. Paidós. Barcelona.

K. Kaliyaperumal, I. E. (2016). Community Ophthalmology. Obtenido de Guideline for Conducting a Knowledge, Attitude and Practice (KAP) Study: http://wwww.birds. cornell.edu/citscitoolkit/toolkit/steps/effects/resource-folder/ Guideline\%20for\%20Conducting\%20a\%20KAP\%20 Study\%20(PDF).pdf
Mora, M. (2002). La teoría de las Representaciones Sociales de Serge Moscovici. Recuperado de Athenea Digital: http://www.raco.cat/index.php/Athenea/article/viewFile/34106/33945

Moscovici, S. (1979). El Psiconálisis su Imágen y su Público. Huemul. Buenos Aires.

Páez, D. (1987). Características, funciones y proceso de formación de las representaciones sociales. En: D. P. colaboradores, Pensamiento, individuo y sociedad. Cognición y representación social. (p. 297-317). Fundamentos. Madrid.

Perera, M. (1999). A propósito de las representaciones sociales: apuntes teóricos, trayectoria y actualidad. Informe de Investigación. Centro de Investigaciones Psicológicas y Sociológicas. La Habana.

Portocarrero, R. (2016). Coordinador Sistema de Información Unicatólica. (E. Latorre, Entrevistador)

Rodriguez, O. (2001). Representación social del alcoholismo. Trabajo de Diploma. Universidad de la Habana. La Habana.

World Health Organization. (2016). Advocacy, communication and social mobilization for TB control. A GUIDE TO DEVELOPING KNOWLEDGE, ATTITUDE AND PRACTICE SURVEYS. Recuperado de http://apps.who.int/iris/bitstre am/10665/43790/1/9789241596176 eng.pdfWorld 110665/43790/1/9789241596176_eng.pdf 